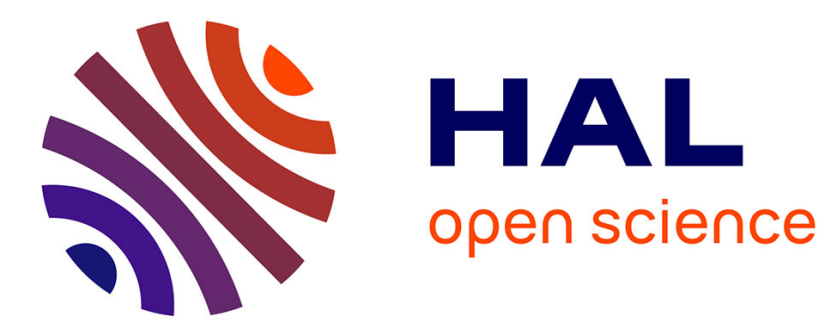

\title{
2D Control Problem and TVD-Particle Method for Water Treatment System
}

Mohammed Louaked, Abdelkader Saïdi

\section{To cite this version:}

Mohammed Louaked, Abdelkader Saïdi. 2D Control Problem and TVD-Particle Method for Water Treatment System. 2011. hal-00579797

\section{HAL Id: hal-00579797 \\ https://hal.science/hal-00579797}

Preprint submitted on 25 Mar 2011

HAL is a multi-disciplinary open access archive for the deposit and dissemination of scientific research documents, whether they are published or not. The documents may come from teaching and research institutions in France or abroad, or from public or private research centers.
L'archive ouverte pluridisciplinaire HAL, est destinée au dépôt et à la diffusion de documents scientifiques de niveau recherche, publiés ou non, émanant des établissements d'enseignement et de recherche français ou étrangers, des laboratoires publics ou privés. 


\title{
2D Control Problem and TVD-Particle Method for Water Treatment System
}

\author{
Mohammed Louaked ${ }^{*}$ and Abdelkader Saïdi ${ }^{\dagger}$
}

\begin{abstract}
This work consists on the study of an optimal control problem relating to the water pollution. We analyze various questions: existence, uniqueness, control and the regularized formulation of the initial pointwise control problem. We propose also an implementation of an hybrid numerical scheme associated with an algorithm of descent.
\end{abstract}

\section{Introduction}

The improvement of the environmental conditions became, these last years, a major issue and in particular the safeguarding of the coastal regions. In this context, we need to predict some parameters, reflecting the quality of the aquatic media, and related to the propagation of the bacteria responsible for the infections transmitted by water or dependant on the consumption of some sea fruits.

To achieve this objective, we mainly address the evolution of waste water concentration based on some convection-reaction-diffusion equations with a pointwise source term modeling the action of the outlets where the convection term is deduced from the coupling with the shallow water system.

To improve the water quality, we introduce a control action at the points source by minimizing the deviation of fecal coliform distribution from standard value.

We firstly, analyze the existence and uniqueness of solution of the transport problem with a source term of Dirac type. For numerical reasons, we regularize the initial problem. We study then the regularized problem and show the convergence of the regular solutions towards that of the initial problem.

The simulation of the transport of biological systems are done by an hybrid numerical procedure combining particle method and finite difference technique.

The control problem is treated by a minimizing algorithm, where the gradient of the cost function is evaluated by adjoint techniques and a gradient type method as an iterative solution of the discrete control problem is chosen.

\footnotetext{
*Laboratoire de Mathématiques Nicolas Oresme, Université de Caen, Campus II, Bd. Maréchal Juin, BP. 5186, 14032 Caen, France, email: louaked@math.unicaen.fr

${ }^{\dagger}$ Institut de Recherche Mathématique Avancée, Université de Strasbourg, 7 rue René Descartes, F-67084 Strasbourg, France, email: saidi@math.unistra.fr
} 
The structure of the paper is the following. We start by recalling the contaminant transport equation and the shallow water system. Next, we analyze the water quality problem and give some uniqueness, existence and regularity results concerning the state equation. After, we present the control problem and investigate the regularized version with an aim of numerical approximation. Finally, we provide numerical test cases.

\section{Contaminant Transport Modeling}

The model describing the evolution of water quality is given by an convection, reaction, diffusion equation where the concentration is solution of the following problem

$$
\frac{\partial C}{\partial t}+\vec{u} . \nabla C-\nu \Delta C=-a_{0} C+\sum_{j=1}^{n} f_{j}(t) \delta_{P_{j}} \quad Q=(0, T) \times \Omega,
$$

with initial and boundary conditions

$$
\begin{gathered}
C(0, x)=C_{0}(x) \quad \text { in } \quad \Omega, \\
\frac{\partial C}{\partial n}(t, x)=0, \quad \text { in } \quad \Sigma=\Gamma \times(0, T),
\end{gathered}
$$

in which $C$ is the concentration of a pollutant, $\vec{u}$ is the external velocity, $\nu>0$ is the dispersion parameter, $a_{0}$ is the strictly positive coefficient for internal source corresponding to a reactive term, $T$ is a specified positive number representing a time horizon and $f_{j}(t)$ is the mass flow rate at a discharge point $P_{j}$ at time $t, \delta_{P_{j}}(x)=\delta\left(x-P_{j}\right)$ is a Dirac measure centred on fixed point $P_{j}$.

The velocity field $\vec{u}=(u, v)$ is related to the water elevation by the shallow water equations (SWE)

$$
\frac{\partial U}{\partial t}+\frac{\partial F(U)}{\partial x}+\frac{\partial G(U)}{\partial y}=S(U)
$$

Where

$$
U=[h, h u, h v]^{T},
$$

denotes the solution vector, and :

$$
\begin{aligned}
& F(U)=\left[h u, h u^{2}+\frac{1}{2} g h^{2}, u v h\right]^{T}, \\
& G(U)=\left[h v, u v h, h v^{2}+\frac{1}{2} g h^{2}\right]^{T},
\end{aligned}
$$

are the vector-valued flux functions. Where $h, h u$ and $h v$, respectively, denote the height of water column and the discharge across the channel section, $\bar{\nu}$ the diffusion coefficient and $g$ is the gravitational acceleration. The source term $S(U)$ contains variable depth, bottom friction

effects and also wind actions. An appropriate boundary and initial conditions are required to solve the SWE system.

The optimal control problem consists of finding a control $f^{*}$ that minimizes a cost functional

$$
J(f)=\Phi(C(f))+\Psi(\|f\|),
$$

where $C($.$) is the state satisfying constraints (1)-(2)-(3) and corresponding to control f()=$. $\left(f_{j}(.)\right)_{j=1 \ldots n}$. The functions $\Phi$ and $\Psi$ are chosen according to the topology of the state space where $C($.$) is considered.$ 


\section{Analysis of the Water Quality Problem}

Starting from the state equation described in the previous section and let us first assume the hypothesis :

H(3.1): The velocity in the boundaries satisfies $\vec{u}(t, x)=0$ in $(0, T) \times \Gamma$.

H(3.2): The function $\vec{u}$ belongs to $L^{\infty}(\Omega \times(0, T)), C_{0} \in L^{2}(\Omega)$ and $f_{j} \in L^{2}(0, T)$ for $j=1 \ldots n$.

Let us multiply equation (1) by a test function $\Phi$, integrate by part and combine conditions (3) and $\mathbf{H}(\mathbf{3 . 1})$, we obtain:

$\left[\int_{\Omega} C(t, x) \Phi(t, x) d x\right]_{0}^{T}+\int_{Q} C\left(-\Phi_{t}-\nabla \cdot(\vec{u} \Phi)-\nu \Delta \Phi+a_{0} \Phi\right) d t d x+\nu \int_{\Sigma} C \frac{\partial \Phi}{\partial n} d t d x=\sum_{j=1}^{n} \int_{0}^{T} f_{j}(t) \Phi\left(t, P_{j}\right) d t$.

For a given $\Psi \in L^{2}(Q)$ we define the adjoint state equation by :

$$
\left\{\begin{array}{lll}
-\Phi_{t}-\nabla(\vec{u} \Phi)-\nu \Delta \Phi+a_{0} \Phi=\Psi & \text { in } & Q, \\
\Phi(T, x)=0 & \text { in } \quad & \Omega, \\
\nu \frac{\partial \Phi}{\partial n}(t, x)=0 & \text { in } & (0, T) \times \partial \Omega .
\end{array}\right.
$$

Remark 1 The weak formulation of the state equation (9) with the use of adjoint problem (10) is known as transposition method and can be formulated as [7]:

Find $C \in L^{2}(Q)$ such that:

$$
\int_{Q} C(t, x) \Psi(t, x) d t d x=\sum_{j=1}^{n} \int_{0}^{T} f_{j}(t) \Phi\left(t, P_{j}\right) d t+\int_{\Omega} C_{0}(x) \Phi(0, x) d x
$$

Where $Q=(0, T) \times \Omega$ and $\Phi$ is a solution of the adjoint state equation (10).

For $\Psi$ in $L^{2}(Q)$ the solution $\Phi$ of (10) is in $L^{2}\left(0, T ; H^{2}(\Omega)\right)$, since $\Omega \subset \mathbb{R}^{2}$ we have the inclusion $H^{2}(\Omega) \subset C(\Omega)$ space of continuous fonction on $\Omega$ such that $\Phi\left(t, P_{j}\right)$ is in $L^{2}(0, T)$ and depends continuously on $\Psi \in L^{2}(Q)$ due to the adjoint problem (10). The right hand side of the weak formulation (11) $: l(\Psi)=\sum_{j=1}^{n} \int_{0}^{T} f_{j}(t) \Phi\left(t, P_{j}\right) d t+\int_{\Omega} C_{0}(x) \Phi(0, x) d x$ is a linear continuous form on $L^{2}(Q)$. As a consequence, we have the following result :

Theorem 3.1 Under the hypothesis $\mathbf{H ( 3 . 1 )}$ and $\mathbf{H}(\mathbf{3 . 2})$ there is a unique solution $C \in L^{2}(Q)$ of problem (11).

\section{Analysis of the Control Problem}

\subsection{Performance function and gradient computation}

To solve the optimal control problem, we have to find a mass flow rates $f^{*} \in \mathcal{G}_{a d}$ at a discharge points $P_{j}, j=1 \ldots n$ minimizing the concentration deviation of a pollutant distribution $C$ from 
standard value $C_{d}$ :

$$
J(f)=\frac{1}{2} \int_{Q}\left(C(t, x)-C_{d}(t, x)\right)^{2} d x d t+\sum_{j=1}^{n} \int_{0}^{T} \frac{\mu_{j}}{2}\left(f_{j}(t)\right)^{2} d t .
$$

In the weighted $L^{2}$ norm penalization term, $\mu_{j}$ is a small positive parameter. The vector control $f(t)=\left(f_{j}(t)\right)_{j=1 \ldots n}$ belongs to a set of admissible controls $\mathcal{G}_{a d}=\left(L^{2}(0, T)\right)^{n}$.

In what follows, we treat the question of existence of an optimal control $f^{*}$ and give the expression of the gradient of the cost function, from which one can set up a scheme to approximate the optimal control.

Theorem 4.1 The above control problem (1) and (12) admits a unique solution $f \in\left(L^{2}(0, T)\right)^{n}$ and the gradient of the cost function is identified as

$$
\nabla J(f)_{j}=\mu_{j} f_{j}(t)+P\left(t, P_{j}\right) \quad j=1, \ldots, n .
$$

Where the adjoint state function $P$ satisfies the following form :

$$
\left\{\begin{array}{lll}
-\frac{\partial P}{\partial t}-\nabla \cdot(\vec{u} P)-\nu \Delta P+a_{0} P=C(t, x)-C_{d}(t, x) & \text { in } & Q, \\
P(T, x)=0 & \text { in } \quad & \Omega, \\
\frac{\partial P}{\partial n}(t, x)=0 & \text { in } & (0, T) \times \Gamma .
\end{array}\right.
$$

Proof.- For clearness reasons, we present our proof into two steps.

Step I: Existence of a minimum

First of all, the operator $f=\left(f_{j}\right)_{j=1 \ldots n} \rightarrow C(f)$ is an affine function from $\left(L^{2}(0, T)\right)^{n}$ to $L^{2}(Q)$. One can set the functional to be optimized as :

$$
\begin{aligned}
J(f) & =\frac{1}{2}\left\|C(f)-C_{d}\right\|_{L^{2}(Q)}^{2}+\sum_{i=1}^{n} \frac{\mu_{j}}{2}\left\|f_{j}\right\|_{L^{2}(0, T)}^{2} \\
& =\frac{1}{2}\left\|(C(f)-C(0))+\left(C(0)-C_{d}\right)\right\|_{L^{2}(Q)}^{2}+\sum_{j=1}^{n} \frac{\mu_{j}}{2}\left\|f_{j}\right\|_{L^{2}(0, T)}^{2} \\
& =\frac{1}{2} b(f, f)-2 L(f)+\left\|C_{d}-C(0)\right\|_{L^{2}(Q)}^{2} .
\end{aligned}
$$

where $b$ is a symmetric bilinear form over $\left(L^{2}(0, T)\right)^{n} \times\left(L^{2}(0, T)\right)^{n}$ defined by

$$
b(f, g)=(C(f)-C(0), C(g)-C(0))_{L^{2}(Q)}+\sum_{j=1}^{n} \frac{\mu_{j}}{2}\left(f_{j}, g_{j}\right)_{L^{2}(0, T)},
$$

and $L$ is a bounded operator on $\left(L^{2}(0, T)\right)^{n}$ given by

$$
L(f)=\left(C_{d}-C(0), C(f)-C(0)\right)_{L^{2}(Q)} .
$$

The form $b$ is $\left(L^{2}(0, T)\right)^{n}$-continuous and coercive by

$$
b(f, f) \geq \frac{\mu}{2}\|f\|_{\left(L^{2}(0, T)\right)^{n}}^{2} \quad \text { with } \quad \mu=\min _{j=1 \ldots n} \mu_{j}
$$


Hence, by Lax-Milgram theorem, one can conclude that $J$ admits a unique optimal solution.

Step II: Gradient identification

Since $\nabla J(f) \in\left(L^{2}(0, T)\right)^{n}$, we have :

$$
\bar{\delta} J(f)=\sum_{j=1}^{n} \int_{0}^{T} \nabla J(f)_{j} \bar{\delta} f_{j} d t,
$$

where $\bar{\delta} J$ is the differential of $J$. It also follows from (1) and (12) that the differential satisfies

$$
\bar{\delta} J(f)=\int_{Q}\left(C-C_{d}\right) \bar{\delta} C d x d t+\sum_{i=1}^{n} \mu_{j} \int_{0}^{T} f_{j} \bar{\delta} f_{j} d t
$$

where $\bar{\delta} C$ solves the linearized convection-diffusion equation :

$$
\begin{array}{ll}
\frac{\partial \bar{\delta} C}{\partial t}+\vec{u} \nabla \bar{\delta} C-\nu \Delta \bar{\delta} C+a_{0} \bar{\delta} C=\sum_{j=1}^{n} \bar{\delta} f_{j} \delta_{P_{j}} & \text { in } \quad Q, \\
\bar{\delta} C(0, x)=0 & \text { in } \quad \Omega, \\
\frac{\partial \bar{\delta} C}{\partial n}(t, x)=0, & \text { in } \quad \Sigma=\Gamma \times(0, T) .
\end{array}
$$

By introducing the adjoint state $P=P(t, x)$ and multiplying both sides of the differential equation in (21) by $P$ and then integrating in both space and time we get :

$$
\begin{aligned}
\int_{Q} \frac{\partial \bar{\delta} C}{\partial t} P d x d t+\int_{Q} \vec{u} \nabla \bar{\delta} C P d x d t-\nu \int_{Q} \Delta \bar{\delta} C P d x d t & +\int_{Q} a_{0} \bar{\delta} C P d x d t \\
& =\sum_{j=1}^{n} \int_{0}^{T} \bar{\delta} f_{j}(t) P\left(t, P_{j}\right) d t
\end{aligned}
$$

If a suitable integration by parts is performed, in both space and time, by the help of the initial and boundary conditions of problem (21) and using hypothesis $\mathbf{H}(\mathbf{3 . 1})$, we find :

$$
\begin{aligned}
\int_{\Omega} \bar{\delta} C(T, x) P(T, x) d x+\int_{Q} \bar{\delta} C\left(-\frac{\partial P}{\partial t}-\nabla \cdot(\vec{u} P)-\nu \Delta P+a_{0} P\right) d t d x & +\nu \int_{\Sigma} \bar{\delta} C(t, x) \frac{\partial P}{\partial n}(t, x) d t d x \\
& =\sum_{j=1}^{n} \int_{0}^{T} \bar{\delta} f_{j}(t) P\left(t, P_{j}\right) d t
\end{aligned}
$$

By requiring the adjoint state function $P$ to satisfy the following form :

$$
\begin{cases}-\frac{\partial P}{\partial t}-\nabla \cdot(\vec{u} P)-\nu \Delta P+a_{0} P=C-C_{d} & \text { in } \quad Q, \\ P(T, x)=0 & \text { in } \quad \Omega, \\ \nu \frac{\partial P}{\partial n}(t, x)=0 & \text { in } \quad \Sigma=\Gamma \times(0, T) .\end{cases}
$$

Then the gradient of the cost function is simply represented by :

$$
\nabla J(f)=\left(\nabla J(f)_{j}\right)_{j=1 \ldots n} \quad \text { with : } \quad \nabla J(f)_{j}=P\left(t, P_{j}\right)+\mu_{j} f_{j}
$$

The expression (13) of the gradient $\nabla J$, using the adjoint problem (14), is directly exploited in the algorithm of descent to achieve the optimal control. 


\section{Regularized Control Problem}

For numerical reasons, a regularized version of the control problem (1) is suitable

$$
\frac{\partial C^{\varepsilon}}{\partial t}+u \nabla C^{\varepsilon}-\nu \Delta C^{\varepsilon}=-a_{0} C^{\varepsilon}+\sum_{j=1}^{n} f_{j}(t) \rho_{\varepsilon}^{j}(x) \quad \text { in } \quad Q .
$$

with initial and boundary conditions

$$
\begin{gathered}
C^{\varepsilon}(0, x)=C_{0}(x) \quad \text { in } \quad \Omega, \\
\frac{\partial C^{\varepsilon}}{\partial n}(t, x)=0, \quad \text { in } \quad(0, T) \times \Gamma .
\end{gathered}
$$

The Dirac measure $\delta_{P_{j}}(x)$ is replaced by a standard function. More precisely, we introduce for sufficiently small $\varepsilon>0$ a set functions $\left\{\rho_{\varepsilon}^{j}(x)\right\}_{\varepsilon}$ as a mollifier which have the following property

$$
\exists C_{1}>0 \quad \text { such that } \quad\left\|\delta_{P_{j}}(x)-\rho_{\varepsilon}^{j}\right\|_{L^{2}(\Omega)} \leq C_{1} \varepsilon
$$

The solution to the initial-boundary value problem is then defined as the limit $\varepsilon \downarrow 0$ of the family $\left\{C^{\varepsilon}\right\}_{\varepsilon}$.

We may introduce a similar control analogous to the equation (12) associated to the regularized problem for the following cost function

$$
J_{\varepsilon}(f)=\frac{1}{2} \int_{Q}\left(C^{\varepsilon}(t, x)-C_{d}(t, x)\right)^{2} d x d t+\sum_{j=1}^{n} \frac{\mu_{j}}{2} \int_{0}^{T}\left|f_{j}(t)\right|^{2} d t .
$$

The optimal control $f_{\varepsilon}^{*}$ is defined as a minimum of the cost function $J_{\varepsilon}($.$) .$

Theorem 5.1 Under the conditions $\mathbf{H}(\mathbf{3 . 1})-\mathbf{H}(\mathbf{3 . 2})$, one has the weak convergence

$$
C^{\varepsilon} \rightarrow C \quad \text { in } L^{2}(Q)
$$

Proof.- For a fixed point $d=\left(d_{1}, d_{2}\right) \in \Omega$ and any point $x=\left(x_{1}, x_{2}\right) \in \Omega$ the delta function is given by $\delta(x)=\delta\left(x_{1}\right) \delta\left(x_{2}\right)$ We first construct a mollifier $\left\{\rho_{\varepsilon}(x)\right\}_{\varepsilon}=\rho_{\varepsilon}^{1}\left(x_{1}\right) \rho_{\varepsilon}^{2}\left(x_{2}\right)$ with the property (29), we first start choosing a $\mathcal{C}^{\infty}$ function $\phi$ such that

$$
\int_{I_{\varepsilon}} P(t) \phi(t) d t=P(d) \quad \forall P \in \mathbb{R}_{1}[X]
$$

where $\left.I_{\varepsilon}=\right] d-\varepsilon, d+\varepsilon[$.

Let put

$$
\rho_{\varepsilon}(x)=\left\{\begin{array}{cc}
\varepsilon^{-1} \phi\left(T_{\varepsilon}(x)\right) & \text { if } x \in I_{\varepsilon} \\
0 & \text { if not. }
\end{array}\right.
$$

Here $T_{\varepsilon}: x \rightarrow \frac{x-d}{\varepsilon}$ is the affine transformation from $I_{\varepsilon}$ onto $]-1,1[$.

Taking $\left.\psi \in H_{0}^{1}\right] a, b[$ and by the help of Bramble-Hilbert lemma, there exist a polynomial $P \in \mathbb{R}_{1}[X]$ such that :

$$
\begin{gathered}
\|\psi-P\|_{L^{2}\left(I_{\varepsilon}\right)} \leq C_{2} \varepsilon\|\psi\|_{H^{1}\left(I_{\varepsilon}\right)}, \\
\|\psi-P\|_{L^{\infty}\left(I_{\varepsilon}\right)} \leq C_{2} \varepsilon^{\frac{1}{2}}\|\psi\|_{H^{1}\left(I_{\varepsilon}\right)} .
\end{gathered}
$$


Since

$$
\begin{aligned}
\left\langle\delta_{d}(x)-\rho_{\varepsilon}, \psi\right\rangle & \\
& =\left\langle\delta_{d}(x), \psi\right\rangle-\left\langle\rho_{\varepsilon}, P\right\rangle-\left\langle\rho_{\varepsilon}, \psi-P\right\rangle \\
& =\psi(d)-P(d)-\left\langle\rho_{\varepsilon}, \psi-P\right\rangle .
\end{aligned}
$$

We conclude that

$$
\left|\left\langle\delta_{d}(x)-\rho_{\varepsilon}, \psi\right\rangle\right| \leq C_{3} \varepsilon^{\frac{1}{2}}\|\psi\|_{V}
$$

and also again

$$
\left\|\delta_{d}(x)-\rho_{\varepsilon}\right\|_{V^{*}} \leq C_{3} \varepsilon^{\frac{1}{2}} \quad \text { as } \quad \varepsilon \longrightarrow 0 .
$$

The difference $D^{\varepsilon}=C-C^{\varepsilon}$ is in $L^{2}(Q)$ and is solution of the problem :

$$
\frac{\partial D^{\varepsilon}}{\partial t}+u \nabla D^{\varepsilon}-\nu \Delta D^{\varepsilon}+a_{0} D^{\varepsilon}=\sum_{j=1}^{n} f_{j}(t)\left(\delta_{P_{j}}(x)-\rho_{\varepsilon}^{j}(x)\right) \quad \text { in } \quad Q,
$$

with initial and boundary conditions :

$$
\begin{gathered}
D^{\varepsilon}(0, x)=0 \quad \text { in } \quad \Omega, \\
\frac{\partial D^{\varepsilon}}{\partial n}(t, x)=0, \quad \text { in } \quad \Sigma=\Gamma \times(0, T) .
\end{gathered}
$$

We multiply (36) by a test function $\Phi$ and integrating by part we have due to (37), (38) we obtain :

$$
\begin{aligned}
& \int_{\Omega} D^{\varepsilon}(T, x) \Phi(T, x) d x+\int_{Q} D^{\varepsilon}\left(-\frac{\partial \Phi}{\partial t}-\nabla \cdot(u \Phi)-\nu \Delta \Phi+a_{0} \Phi\right) d t d x+\nu \int_{\Sigma} D^{\varepsilon}(t, x) \frac{\partial \Phi}{\partial n}(t, x) d t d x \\
& =\sum_{j=1}^{n} \int_{Q} f_{j}(t)\left(\delta_{P_{j}}(x)-\rho_{\varepsilon}^{j}(x)\right) \Phi(t, x) d t d x
\end{aligned}
$$

for a given $\Psi \in L^{2}(Q)$ we define the function $\Phi$ as a solution of the problem (10) and then we have :

$$
\int_{Q} D^{\varepsilon} \Psi d t d x=\sum_{j=1}^{n} \int_{Q} f_{j}(t)\left(\delta_{P_{j}}(x)-\rho_{\varepsilon}^{j}(x)\right) \Phi(t, x) d t d x
$$

using the fact that $\Phi$ is solution of (10) we have :

$$
\begin{gathered}
\int_{Q}\left(C-C^{\varepsilon}\right) \Psi d t d x \leq C_{0}\left(\sum_{j=1}^{n}\left\|f_{j}(t)\left(\delta_{P_{j}}(x)-\rho_{\varepsilon}^{j}(x)\right)\right\|_{L^{2}(Q)}\|\Psi\|_{L^{2}(Q)}\right) \longrightarrow 0 \quad \text { when } \quad \varepsilon \longrightarrow 0 \\
f_{j}(t) \rho_{\varepsilon}^{j}\left(x-P_{j}\right) \longrightarrow f_{j}(t) \delta_{P_{j}}(x) \quad \text { in } \quad L^{2}(Q),
\end{gathered}
$$

we deduce the weak convergence in $L^{2}(Q)$.

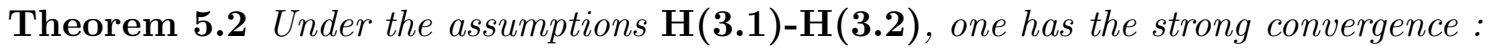

$$
C^{\varepsilon} \longrightarrow C \quad \text { in } L^{2}(Q)
$$


Proof.-

We multiplay equation (36) by a test function $P^{\varepsilon}$ and integrate by part over $Q$ we obtain using conditions (37), (38) :

$$
\begin{aligned}
& \int_{\Omega} D^{\varepsilon}(T, x) P^{\varepsilon}(T, x) d x+\int_{Q} D^{\varepsilon}\left(-\frac{\partial P^{\varepsilon}}{\partial t}-\nabla \cdot\left(u P^{\varepsilon}\right)-\nu \Delta P^{\varepsilon}+a_{0} P^{\varepsilon}\right) d t d x+\nu \int_{\Sigma} D^{\varepsilon}(t, x) \frac{\partial P^{\varepsilon}}{\partial n}(t, x) d t d x \\
& =\sum_{j=1}^{n} \int_{Q} f_{j}(t)\left(\delta_{P_{j}}(x)-\rho_{\varepsilon}^{j}(x)\right) P^{\varepsilon}(t, x) d t d x
\end{aligned}
$$

Let us know define $P^{\varepsilon}$ as the solution of the following adjoint problem :

$$
\begin{cases}-\frac{\partial P^{\varepsilon}}{\partial t}-\nabla \cdot\left(u P^{\varepsilon}\right)-\nu \Delta P^{\varepsilon}+a_{0} P^{\varepsilon}=C-C^{\varepsilon} & \text { in } \quad Q, \\ P^{\varepsilon}(T, x)=0 & \text { in } \quad \Omega, \\ \nu \frac{\partial P^{\varepsilon}}{\partial n}(t, x)=0 & \text { in } \quad \Sigma=\Gamma \times(0, T) .\end{cases}
$$

We obtain from (45) and formal week formulation (44) :

$$
\int_{Q} D^{\varepsilon}\left(C-C^{\varepsilon}\right) d t d x=\sum_{j=1}^{n} \int_{Q} f_{j}(t)\left(\delta_{P_{j}}(x)-\rho_{\varepsilon}^{j}(x)\right) P^{\varepsilon}(t, x) d t d x
$$

We have then the existence and uniqueness of a solution of (46) with the transposition method (ref) and for $C-C^{\varepsilon}=D^{\varepsilon} \in L^{2}(Q)$ the solution of (45) is in $L^{2}\left(0, T ; H^{2}(\Omega)\right)$ and depends continuously from $D^{\varepsilon}$. As a consequence of that there exist a constante $C_{0}$ such that :

$$
\left\|C-C^{\varepsilon}\right\|_{L^{2}(Q)}^{2}=\int_{Q}\left(D^{\varepsilon}\right)^{2} d t d x \leq C_{0}\left(\left\|f_{j}(t)\left(\delta_{P_{j}}(x)-\rho_{\varepsilon}^{j}(x)\right)\right\|_{L^{2}(Q)}\left\|C-C^{\varepsilon}\right\|_{L^{2}(Q)}\right.
$$

and then we have :

$$
\left\|C-C^{\varepsilon}\right\|_{L^{2}(Q)} \leq C_{0}\left(\sum_{j=1}^{n}\left\|f_{j}(t)\left(\delta_{P_{j}}(x)-\rho_{\varepsilon}^{j}(x)\right)\right\|_{L^{2}(Q)} \longrightarrow 0 \quad \text { when } \quad \varepsilon \longrightarrow 0\right.
$$

The following result establishes the convergence of the regularized minima towards that of the initial problem.

Theorem 5.3 Let us set

$$
j_{\varepsilon}=J_{\varepsilon}\left(f_{\varepsilon}\right)=\operatorname{In} f_{f \in \mathcal{G}_{a d}} J_{\varepsilon}(f) \quad \text { and } \quad j=J\left(f^{\star}\right)=\operatorname{In} f_{f \in \mathcal{G}_{a d}} J(f) .
$$

We assume that $\mathbf{H}(\mathbf{3 . 1}) \mathbf{- H}(\mathbf{3 . 2})$ hold. Then as $\varepsilon \longrightarrow 0$, we have

$$
\begin{aligned}
& j_{\varepsilon} \longrightarrow j \quad \text { in } \quad \mathbb{R} \text {. } \\
& f_{\varepsilon}^{\star} \longrightarrow f^{\star} \quad \text { in } \quad \mathcal{G}_{a d} .
\end{aligned}
$$


Proof.- Since $C^{\varepsilon} \longrightarrow C$ in $L^{2}(Q)$, we have

$$
J_{\varepsilon}\left(f^{\star}\right) \longrightarrow J\left(f^{\star}\right) \quad \forall f^{\star} \text { fixed in } \mathcal{G}_{a d} .
$$

In particular as $\varepsilon \downarrow 0$

$$
j_{\varepsilon} \leq J_{\varepsilon}\left(f^{\star}\right) \longrightarrow{ }_{\varepsilon \downarrow 0} J\left(f^{\star}\right)=j
$$

giving us

$$
\limsup j_{\varepsilon} \leq j
$$

but

$$
j_{\varepsilon}=J_{\varepsilon}\left(f_{\varepsilon}\right) \geq \sum_{j=1}^{n} \frac{\mu_{j}}{2} \int_{0}^{T}\left(f_{\varepsilon}\right)_{j}^{2} d t \geq \frac{\mu}{2}\left\|f_{\varepsilon}\right\|_{\left(L^{2}(0, T)^{n}\right.}^{2} \quad \text { with } \quad \mu=\min _{j=1 \ldots n} \mu_{j}
$$

we may extract a subsequence $f_{j_{\varepsilon}}$, denoted again by $f_{\varepsilon}$, such that

$$
f_{\varepsilon} \rightarrow g \quad \text { in } \quad \mathcal{G}_{a d}
$$

and by virtue of theorem 5.1 ( a faire .... )

$$
\liminf J\left(f_{\varepsilon}\right) \geq J(g)
$$

But as

$$
\lim _{\varepsilon \rightarrow 0}\left(J\left(f_{\varepsilon}\right)-J_{\varepsilon}\left(f_{\varepsilon}\right)\right)=0
$$

we also have

$$
\liminf J\left(f_{\varepsilon}\right) \leq \liminf J_{\varepsilon}\left(f_{\varepsilon}\right)
$$

from which we obtain

$$
\liminf J\left(f_{\varepsilon}\right) \geq J(g)
$$

By the virtue of the inequalities $\lim \sup j_{\varepsilon} \leq j, \quad J(g) \leq \liminf j_{\varepsilon} \leq j$ and by the uniqueness of the minimum, we get

$$
g=f^{\star}
$$

It follows

$$
j \leq \liminf j_{\varepsilon} \leq j
$$

and hence

$$
j_{\varepsilon} \rightarrow j \quad \text { because } \quad j \geq \lim \sup j_{\varepsilon}
$$

consequently

$$
j_{\varepsilon} \longrightarrow j
$$

It remains to prove the strong convergence of $f_{\varepsilon}$ towards $f^{\star}$. For such purpose we combine the facts

$$
\begin{aligned}
& J_{\varepsilon}\left(f_{\varepsilon}\right) \\
& =\frac{1}{2} \int_{Q}\left(C^{\varepsilon}(t, x)-C_{d}(t, x)\right)^{2} d x d t+\sum_{j=1}^{n} \frac{\mu_{j}}{2} \int_{0}^{T}\left(f_{\varepsilon}\right)_{j}^{2}(t) d t \\
& =\frac{1}{2} \int_{Q}\left(C^{\varepsilon}(t, x)\right)^{2} d x d t+\sum_{j=1}^{n} \frac{\mu_{j}}{2} \int_{0}^{T}\left(f_{\varepsilon}\right)_{j}^{2}(t) d t-\int_{Q} C^{\varepsilon}(t, x) C_{d}(t, x) d x d t+\frac{1}{2} \int_{Q}\left(C_{d}(t, x)\right)^{2} d x d t \\
& \longrightarrow J\left(f^{\star}\right) \quad \text { as } \quad \varepsilon \rightarrow 0,
\end{aligned}
$$


and

$$
J_{\varepsilon}(0) \longrightarrow J(0)
$$

with the weak convergence of $f_{\varepsilon}$ towards $f^{\star}$ (see equations (53-(54)) and the convergence (31), we obtain first

$$
b\left(f_{\varepsilon}, f_{\varepsilon}\right) \longrightarrow b\left(f^{\star}, f^{\star}\right),
$$

and since the bilinear form $b(g, g)$ defined by (16) is equivalent to $\|g\|_{\left(L^{2}(0, T)\right)^{n}}^{2}$, finally by virtue of the coercivity (18) we have the convergence $f_{\varepsilon} \longrightarrow f^{\star}$ in $\mathcal{G}_{a d}$.

\section{Numerical Approximation}

For the numerical resolution, a hybrid method seems to be well adapted. More precisely, a Lagrangian procedure is used to solve both the primal problem (concentration of pollutant) and its dual (adjoint state). On the other hand, a finite difference approximation (total variation diminishing (TVD)) for the shallow water system[9] is preferred. An algorithm of descent allows to estimate the optimal control where in each step, the resolution of the adjoint problem gives an access to the expression of the gradient.

In what follows, we give details concerning the solution for the $1 \mathrm{D}$ problem and for the $2 \mathrm{D}$ system, we use the well-known dimension by dimension technique.

\subsection{The TVD Scheme}

An explicit conservative difference scheme

$$
U_{j}^{n+1}=U_{j}^{n}-\lambda\left(\hat{F}_{i+\frac{1}{2}}^{n}-\hat{F}_{i-\frac{1}{2}}^{n}\right),
$$

is serving as consistent approximation to the $1 \mathrm{D}$ shallow water system

$$
U_{t}+f(U)_{x}=0
$$

Consider a uniform grid and let the grid spacing be denoted by $\Delta x$ such that $x_{i}=i \Delta x$ and $\Delta t$ be the time step. Let $a^{l}$ be the eigenvalues of $A$, matrix jacobian of $F$, and $R$ is the matrix whose columns are right eigenvectors of $A$. The quantities $a^{l}, R$, evaluated at $U_{i+\frac{1}{2}}$ are respectively denoted by $a_{i+\frac{1}{2}}^{l}$ and $R_{i+\frac{1}{2}}$.

The difference of the characteristic variables in the local $x$-direction is defined by

$$
\alpha_{i+\frac{1}{2}}=R_{i+\frac{1}{2}}^{-1}\left(U_{i+1}-U_{i}\right) .
$$

The numerical flux $\hat{F}_{i+\frac{1}{2}}$ with a diffusive term can be expressed as

$$
\hat{F}_{i+\frac{1}{2}}^{n}=\frac{1}{2}\left[F_{i}^{n}+F_{i+1}^{n}+R_{i+\frac{1}{2}} \Phi_{i+\frac{1}{2}}\right],
$$

where the elements of $\Phi_{i+\frac{1}{2}}$ are given by

$$
\Phi_{i+\frac{1}{2}}^{l}=\frac{1}{2} \sigma\left(a_{i+\frac{1}{2}}^{l}\right)\left(g_{i}^{l}+g_{i+1}^{l}\right)-\Psi\left(a_{i+\frac{1}{2}}^{l}+\gamma_{i+\frac{1}{2}}^{l}\right) \alpha_{i+\frac{1}{2}}^{l},
$$


with

$$
\begin{gathered}
\sigma(z)=\left[\Psi(z)-\frac{\Delta t}{\Delta x}, z^{2}\right] \\
\gamma_{i+\frac{1}{2}}^{l}=\frac{1}{2} \sigma\left(a_{i+\frac{1}{2}}^{l}\right)\left\{\begin{array}{cc}
\frac{\left(g_{i+1}^{l}-g_{i}^{l}\right)}{\alpha_{i+\frac{1}{2}}^{l}}, & \alpha_{i+\frac{1}{2}}^{l} \neq 0 \\
0, & \alpha_{i+\frac{1}{2}}^{l}=0,
\end{array}\right.
\end{gathered}
$$

the entropy correction function is of the form

$$
\Psi(z)=\left\{\begin{array}{cc}
|z| & |z| \leq \varepsilon \\
\frac{\left(z^{2}+\varepsilon^{2}\right)}{2 \varepsilon} & |z| \geq \varepsilon
\end{array}\right.
$$

where $\varepsilon$ is a positive small number.

A compressive limiter,

$$
g_{i}^{l}=S . \max \left[0, \min \left(2\left|\alpha_{i+\frac{1}{2}}^{l}\right|, S . \alpha_{i-\frac{1}{2}}^{l}\right), \min \left(\left|\alpha_{i+\frac{1}{2}}^{l}\right|, 2 S . \alpha_{i-\frac{1}{2}}^{l}\right)\right] ; S=\operatorname{sgn}\left(\alpha_{i+\frac{1}{2}}^{l}\right),
$$

is chosen to deal with the linearly degenerate fields for better resolution of contact discontinuities and a less compressive limiter,

$$
g_{i}^{l}=\operatorname{minmod}\left(\alpha_{i-\frac{1}{2}}^{l}, \alpha_{i+\frac{1}{2}}^{l}\right),
$$

to the genuinely nonlinear fields in order to avoid the steepening problem.

\subsection{Particle Method}

In this section, both regularized and adjoint state are solved with the use of the vortex method. Vortex methods has a grid-free character with an appealing physical interpretation in which the particles carry a concentration of a pollutant and convect with the local flow velocity.

In order to define a numerical method to solve the problem

$$
\begin{aligned}
\frac{\partial C}{\partial t}+u . \nabla C-\nu \Delta C & =\mathcal{L}(t, x) \quad \text { in } \quad(0, T) \times \Omega, \\
C(0, x) & =C_{0}(x) \quad \text { in } \quad \Omega, \\
\frac{\partial C}{\partial n}(t, x) & =0 \quad \text { in } \quad(0, T) \times \Gamma,
\end{aligned}
$$

we use a quadrature rule defined by a set of weighted points $\left(x_{p}^{0}, \alpha_{p}\right)_{p} \in J_{1}$ to provide a highorder approximation to the initial data, where $x_{p}^{0} \in \Omega$ and $\alpha_{p}^{0}>0$.

We have, for any function $\Phi \in C^{0}(\Omega)$

$$
\int_{\Omega} \Phi(x) d x \approx \sum_{p \in J_{1}} \alpha_{p}^{0} \Phi\left(x_{p}^{0}\right) .
$$

The solution $C$ is sought as a linear combination of Dirac distributions

$$
c_{h}(x, t)=\sum_{p \in J_{1}} \alpha_{p}(t) c_{p}(t) \delta_{x_{p}(t)}(x),
$$


where the positions $\left(x_{p}(t)\right)_{p}$ of the particles are defined according to the characteristic curves

$$
\frac{d x_{p}}{d t}(t)=u\left(x_{p}(t), t\right), \quad x_{p}(0)=x_{p}^{0},
$$

and the weights $\left.\left(c_{p}(t)\right)_{p}\right)$ are determined by resolution of the ordinary differential system

$$
\frac{d c_{p} \alpha_{p}}{d t}(t)-\nu \alpha_{p}(t) \Delta_{h} c_{p}(t)=\alpha_{p}(t) \mathcal{L}\left(t, x_{p}(t)\right)
$$

The integral approximation of Laplace's operator is solved by the deterministic Particle Strength Exchange method (PSE)[4]

$$
\Delta_{h}^{\varepsilon} c_{p}(t)=\frac{1}{\varepsilon} \sum_{r \in J_{1}} \alpha_{r}(t)\left(c_{r}(t)-c_{p}(t)\right) \eta_{\varepsilon}\left(x_{p}(t)-x_{r}(t)\right) .
$$

To define a more classical approximation of $C$, we introduce an even compactly supported function $\chi$ on $\mathbb{R}$ such that

$$
\int_{\mathbb{R}} \chi(x) d x=1, \quad \int_{\mathbb{R}} \chi(x) x^{2} d x=2
$$

and let $\chi_{\varepsilon}(x)=\varepsilon^{-1} \chi(x / \varepsilon)$. The more classical approximation $c_{h}^{\varepsilon}$ of the function $C$ than the measure $c_{h}$ is given by

$$
c_{h}^{\varepsilon}(x, t)=\sum_{p \in J} \alpha_{p}(t) c_{p}(t) \chi_{\varepsilon}\left(x-x_{p}(t)\right) .
$$

The resolution of the convection-diffusion equation can be summarized by the resolution of a large system

$$
\left\{\begin{array}{l}
\frac{d x_{p}}{d t}(t)=u\left(t, x_{p}(t)\right) \\
\frac{d c_{p} \alpha_{p}}{d t}(t)-\nu \alpha_{p}(t) \Delta_{h}^{\varepsilon} c_{p}(t)=\alpha_{p}(t) \mathcal{L}\left(t, x_{p}(t)\right) \\
\frac{d \alpha_{p}}{d t}(t)=\alpha_{p}(t) u_{x}\left(t, x_{p}(t)\right) \\
x_{p}(0)=x_{p}^{0}, \quad c_{p}(0)=c_{0}\left(x_{p}^{0}\right) \quad \alpha_{p}(0)=\alpha_{p}^{0}
\end{array}\right.
$$

\subsection{Particle-Mesh interpolation}

The assignment operators, particle strength to the mesh value and the interpolation of the particle values from the mesh, use the moment-conserving $M_{4}^{\prime}$ scheme proposed by Monaghan[13]

$$
M_{4}^{\prime}(x)\left\{\begin{array}{cc}
1-\frac{5}{2}|x|^{2}+\frac{3}{2}|x|^{3}, & |x|<1 \\
\frac{3}{2}(2-|x|)^{2}(1-|x|), & 1<|x|<2 \\
0, & |x|>2 .
\end{array}\right.
$$

\subsection{Optimal control method}

In what follows, we present an algorithm of descent for the control estimation. The method is of gradient type and it is based on the use of an adjoint state given by the theorem 0.2 . 


\subsubsection{Algorithm}

Choose $f^{(0)}(t)$ given in $\left(L^{2}(0, T)\right)^{n}$

0. Compute an approximated solution $C^{(0)}(t, x)$ by solving $(26)$ with $f(t)=f^{(0)}(t)$

Compute an approximated solution $P^{(0)}(t, x)$ by solving (14) with $C=C^{(0)}$

Compute the gradient of descent $G_{j}^{(0)}(t)=\mu_{j} f_{j}^{(0)}(t)+P^{(0)}\left(t, P_{j}\right)$.

For $k=0,1,2, \ldots$

1. Update the control by using the gradient $f^{(k+1)}(t)=f^{(k)}(t)+\rho G^{(k)}(t)$

Compute an approximated solution $C^{(k+1)}(t, x)$ by solving $(26)$ with $f(t)=f^{(k+1)}(t)$

Compute an approximated solution $P^{(k+1)}(t, x)$ by solving (14) with $C=C^{(k+1)}$

Compute the gradient of descent $G_{j}^{(k+1)}(t)=\mu_{j} f_{j}^{(k+1)}(t)+P^{(k+1)}\left(t, P_{j}\right)$

2. If $\left(\frac{\left|G^{(k+1)}\right|}{\left|G^{(k)}\right|}\right)>$ tol then put $f^{(k)}(t)=f^{(k+1)}(t)$, put $G^{(k)}(t)=G^{(k+1)}(t)$ and goto 1 .

end

\subsection{Numerical Tests}

In this section, we present computation results using the hybrid method.

\subsubsection{Peak in the concentration of pollutant}

The hydrodynamic initialization is a dam break on flat bottom [2], due to the fact that this test includes several features of existing issues in the area of marine hydrodynamics and coastal engineering. The source term $S$, in the shallow water system, is equal to zero. There is a peak in the concentration of pollutant in the area $[-0.7,-0.3]$. Computation of the control problem is performed with the following values :

- Spatial domain : $x \in[-1,1]$.

- Physical time : $T=0.24$.

- Discharge point : $d=-0.5$.

- Initial elevation :

$h(0, x)=1$ if $x<0$ else $h(0, x)=0.5$.

- Initial velocity :

$u(0, x)=0 \quad \forall x \in[-1,1]$.

- Initial value for the concentration of pollutant :

$c(0, x)=0.7$ if $x \in[-0.7,0.7]$ else $c(0, x)=0.5$.

- Standard value :

$c_{d}(t, x)=0.44 \quad \forall x \in[-1,1]$.

- Physical parameters :

$a_{0}=1$ and $\nu=0.01$. 
- Computational parameters :

$\Delta x=1 / 128, \mu=0.01$ and $\varepsilon=\Delta x$.

In term of setting, a tolerance of $10^{-5}$ in the gradient algorithm is used as the stopping criterion or we stopped iterating after a fixed number of iterations. As can be seen in Fig 3, the pollutant distribution after convergence is reduced under the desired value.
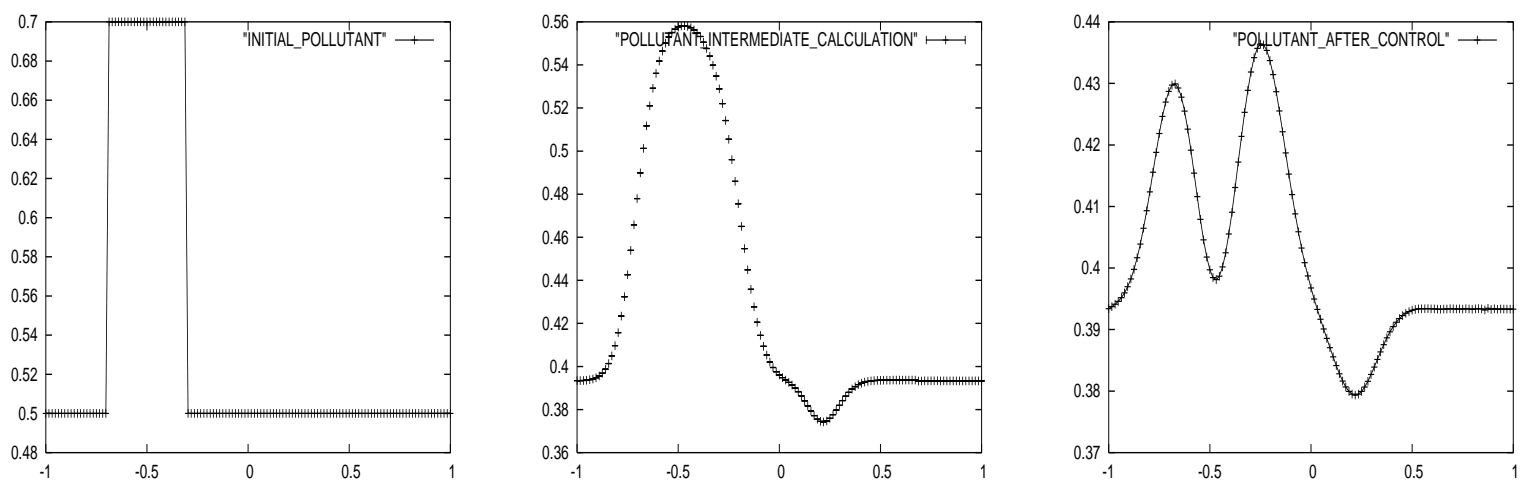

Figure 1: Initial condition for pollutant, pollutant during iterative process, pollutant concentration after convergence at $T=0.24$.

\section{Conclusion}

This work study an environmental control model. The mathematical analysis of different related problems is firstly investigated. After, we treat the numerical part in which we propose an hybrid Particle-TVD method to solve the pollutant transport equations. Our results indicate that the particle strength exchange technique coupled appropriately to a TVD scheme is a robust and an efficient procedure for the direct control concentration pollutant problem.

\section{References}

[1] K. Ammari and A. Saïdi. Pointwise Stabilization of a Hybrid System and Optimal Location of Actuator. Appl. Math. Optimization vol. 56, no. 1, pp 105-130, 2007.

[2] E. Audusse, M. Bristeau., Transport of Pollutant in Shallow Water. A Two Time Steps Kinetic Method. M2AN Math. Model. Numer. Anal. vol. 37, pp 389-416, 2003.

[3] W. Daehyun and A. F. Ghoniem Modified Interpolation Kernels for Treating Diffusion and Remeshing in Vortex Methods. J. Comput. Phys., vol. 213, pp 239-263, 2006.

[4] P. Degond, S. Mas-Gallic., The weighted particle method for convection-diffusion equations. Math. Comput., vol. 53, pp 485-526, 1989.

[5] J. D. Eldredge, A. Leonard and T. Colonius A General Deterministic Treatment of Derivatives in Particle Methods. J. Comput. Phys., vol. 180, pp 686-709, 2002.

[6] J. L. Lions. Contrôle optimal des systèmes gouvernés par des équations aux dérivées partielles. Editions Dunod, 1968. 
[7] J. L. Lions and E. Magenes. Problèmes aux limites non homogènes et applications. Editions Dunod, Vol. 1 et 2, 1968.

[8] M. Louaked, O. Pironneau and S. Mas-Gallic. A Particle in Cell Method for the 2-D Compressible Euler Equations. In Vortex Flow and Related Numerical Methods, p 373-387, Kluwer Academic Publishers, 1993.

[9] M. Louaked and L. Hanich. TVD-Multiresolution Scheme for the Shallow Water equations. Comptes Rendus de l'Académie des Sciences Paris, vol. 331, Série I, pp 745-750, 2000.

[10] M. Louaked, A. Saïdi., Pointwise Control and Particle Analysis for Parabolic Equation. Nonlinear Analysis Series A: Theory Methods\&Applications, vol. 71, pp 2337-2349, 2009.

[11] M. Louaked, A. Saïdi., 2D Control problem for water treatment system. Preprint IRMA, Université de Strasbourg, 2010.

[12] A. Martinez, C. Rodriguez and M. E. Vasquez-Mendez. A Control Problem Related to Wastewater Treatment. Comptes Rendus de l'Académie des Sciences Paris, vol. 328, Série I, pp 35-40, 1999.

[13] J. J. Monaghan Extrapolating B splines for interpolation. J. Comput. Phys., vol. 200, pp 253-262, 2002.

[14] P. A. Raviart. An Analysis of Particle Methods. CIME Course Italy 1983. 